\title{
Paradoxical role of high mobility group box 1 in glioma: a suppressor or a promoter?
}

\author{
Richard A. Seidu, ${ }^{1,2}$ Min Wu, ${ }^{1}$ Zhaoliang Su, ${ }^{2}$ Huaxi $\mathrm{Xu}^{2}$ \\ ${ }^{1}$ Department of Neurosurgery, Affiliated Hospital of Jiangsu University (Jiangbin Hospital), Zhenjiang; ${ }^{2}$ Department \\ of Immunology, Jiangsu University, Zhenjiang, China
}

\begin{abstract}
Gliomas represent $60 \%$ of primary intracranial brain tumors and $80 \%$ of all malignant types, with highest morbidity and mortality worldwide. Although glioma has been extensively studied, the molecular mechanisms underlying its pathology remain poorly understood. Clarification of the molecular mechanisms involved in their development and/or treatment resistance is highly required. High mobility group box 1 protein (HMGB1) is a nuclear protein that can also act as an extracellular trigger of inflammation, proliferation and migration, through receptor for advanced glycation end products and toll like receptors in a number of cancers including gliomas. It is known that excessive release of HMGB1 in cancer leads to unlimited replicative potential, ability to develop blood vessels (angiogenesis), evasion of programmed cell death (apoptosis), self-sufficiency in growth signals, insensitivity to inhibitors of growth, inflammation, tissue invasion and metastasis. In this review we explore the mechanisms by which HMGB1 regulates apoptosis and autophagy in glioma. We also looked at how HMGB1 mediates glioma regression and promotes angiogenesis as well as possible signaling pathways with an attempt to provide potential therapeutic targets for the treatment of glioma.
\end{abstract}

Correspondence: Richard A. Seidu, Department of Neurosurgery, Affiliated Hospital of Jiangsu University (Jiangbin Hospital), Zhenjiang, 212001 China; and Department of Immunology, Jiangsu University, 301 Xuefu Road, Zhenjiang, Jiangsu, 212013 China.

Tel.: +86.511.85038140 - Fax: +86.511.85038449.

E-mail: gbepoo@gmail.com ; gbepo@yahoo.com

Key words: HMGB1; glioma; autophagy; apoptosis; macrophages.

Contributions: the authors contributed equally to the manuscript design and writing.

Conflict of interest: the authors declare no potential conflict of interest.

Received for publication: 11 November 2016.

Revision received: 13 February 2017.

Accepted for publication: 27 February 2017.

This work is licensed under a Creative Commons Attribution NonCommercial 4.0 License (CC BY-NC 4.0).

(C) Copyright R.A. Seidu et al., 2017

Licensee PAGEPress, Italy

Oncology Reviews 2017; 11:325

doi:10.4081/oncol.2017.325

\section{Introduction}

Gliomas are primary brain tumors that originates from nerve stromal cells including glial cells, ependymal cells, choroid plexus epithelial cells, and nerve parenchymal cells and noted for their high morbidity, high recurrence, high case-fatality rate (overall 5year survival of only $13 \%$ ), low cure rate and accounts for about $60 \%$ of all intracranial primary brain tumors. ${ }^{1,2}$ Gliomas are malignant tumors with multigene abnormality and their pathogenesis propose to be as a result of activation of proto-oncogene's high-expression and/or deletion as well as inactivation of cancer suppressor gene causing abnormalities in cell signaling conduction path, change in cell cycles, extension of life cycle, defect of apoptosis etc. which leads to ferocious cell proliferation and malignant transformation. ${ }^{3,4}$ High mobility group box 1 protein (HMGB1) was discovered forty years ago in calf thymus and named according to its electrophoretic mobility in polyacrylamide gels. ${ }^{5}$ HMGB1 has been detected in immature cells and a variety of solid tumors. Many researchers have found HMGB1 to be highly expressed in many malignancies including lung cancer, breast cancer, head and neck squamous cell carcinoma, colon cancer, nasopharyngeal carcinoma, ${ }^{6-8}$ and have associated it with their occurrence, invasion, and metastasis. It is still unclear the specific mechanisms governing the biological activities of HMGB1 and gliomas and very few researches have explored the role of HMGB1 in the development of glioma cells. Current studies in molecular biology of tumors are exploring the mechanisms of gliomas occurrence, progress and therapy, such as targeted therapy, which could be a new and effective treatment method. Our review focuses on the mechanisms by which HMGB1 regulates apoptosis and autophagy in glioma. We also looked at how HMGB1 mediates glioma regression and promotes angiogenesis as well as possible signaling pathways with an attempt to provide potential therapeutic targets for the treatment of gliomas.

\section{Structure}

Human HMGB1 is an alarmin encoded by a single gene that is located in chromosome 13q12. It contain four introns and five exons, encoding a 215-aminoacid protein, with a molecular weight of about $30 \mathrm{KD}$ and highly conserved, with an amino acid sequence homology of over $98 \%$ between human and rodents. ${ }^{9,10}$ HMGB1 protein is divided into three domains: two positively charged DNA-binding motifs (boxes A, B) and a C-terminal acidic tail. While the two motifs contains 80-90 amino acid residues and are strongly alkaline, the carboxyl terminus also known as acidic terminal is rich in aspartic and glutamic acids and negatively charged. ${ }^{11}$ 


\section{Function}

HMGB1 binds to DNA with specific structures and affect the structural state of their target sequence and thus participate in the key life activities including the division, differentiation, and maturation of cells, DNA repair, DNA recombination, regulation by steroid hormones, and regulation of gene transcription ${ }^{12,13}$ hence HMGB1 is a DNA binding protein in the nucleus. Outside the cells, HMGB1 acts as typical injury-related molecule when released by necrotic cells or activated immune cells and by interacting with cytokines, chemokines, and growth factors, it regulates various activities of cells. ${ }^{5}$ Excessive secretion of HMGB1 can suppress apoptosis and thus cause the occurrence and development of tumors hence it is an anti-apoptotic protein. ${ }^{14}$ HMGB1 is also associated with the activation of the matrix metalloproteinases, plasminogen system and the migration of adherent cells. ${ }^{15}$ Therefore HMGB1 is a multifunctional protein.

HMGB1 acts as a pro-tumor protein in the extracellular compartment due to its cytokine, chemokine and growth factor activity. It is now clear that certain amino acids in the B box domain are vital for the protein's pro-inflammatory cytokine function, particularly the cysteine amino acid in the position 106 (C106) in the B box domain that is a requisite for HMGB1 to bind to toll like receptor-4 (TLR-4) activating macrophages. ${ }^{16,17}$ HMGB1 activates dendritic cell via the reduced form of C106. HMGB1 has no posttranslational modifications in its completely reduced state. The reduced form is usually the main form in nucleus and cytoplasm in basal conditions and interacts with numerous receptors, such as CXCR4, to promote cell migration. HMGB1 inflammatory ability is prohibited when cysteines are oxidized to sulfonic acids, implying that the function of HMGB1 can be modified via the cysteine redox modifications. ${ }^{16,18}$ HMGB1 is oxidized in the cytoplasm after reactive oxygen species (ROS production). ${ }^{16,19}$ It is well known that the extracellular environment is mainly oxidative during inflammatory processes. The oxidation of HMGB1 acts as a physiological negative feedback that restricts the pro-inflammatory activity of HMGB1 preventing excessive inflammatory damage. ${ }^{16,18}$ After oxidation, sulfonyl HMGB1 has no activity for cell migration or cytokine induction. ${ }^{16,19}$ Oxidative stress appears to be a central regulator of HMGB1's translocation, release and activity in inflammation and cell death although this mechanism is not yet clear. ${ }^{16,20}$ Calcium- or ROS-dependent mechanisms have been confirmed to induce HMGB1 release. ${ }^{13,16}$

\section{HMGB1 regulates apoptosis in glioma}

Zhao and Chen demonstrated that HMGB1 is eminently released under normal conditions and authenticate potential activity of cancer suppressor gene. They indicated that the percentage of apoptotic cells relatively elevated after the exogenous $H M G B 1$ gene is transferred into the CD133 glioma cells with an originally low release, which means that HMGB1 is on the upstream of the regulatory pathway and its stimulation or over-release can promote apoptosis of glioma cells. ${ }^{21}$

Studies have shown that necrotic cells can release HMGB1 into the extracellular environment, ${ }^{22}$ and necrosis is a characteristic feature of malignant gliomas. It is well noted that consistent secretion of HMGB1 enhances the growth and progress of gliomas leading to continues necrosis of the lesions. This was supported by Jing and colleagues who explored the role of $H M G B 1$ gene in the
U251 and U-87MG cells and also concluded that the up-regulated HMGB1 plays a key role in the development of gliomas. ${ }^{23}$ Further study has demonstrated that the degree of secretion of HMGB1 in different pathological grade of gliomas and noted a profound difference between them. The central role of HMGB1 in necrosis and malignancy in glioma is due to an autocrine factor which promotes the growth and migration of tumor cells. ${ }^{24}$

Other authors are of the view that HMGB1 that is released into the extracellular environment may cause surrounding tumor cells to undergo constant proliferation and induce the regeneration of small blood vessels, thus promoting tumor growth. HMGB1 may cause tumorigenesis by disordered gene secretion, resulting in glial cells obtaining a tumor phenotype and resistance to apoptosis. ${ }^{25}$ Research has further indicated that the necrotic tumor cells which secrete HMGB1 accelerates tumor development and infiltration into the surrounding brain tissue hence presents a stronger resistance, which makes it difficult to attain whole resection leading to poor prognosis. ${ }^{26}$ Also, growth and migration cells in gliomas in vitro were suppressed when HMGB1 was inhibited. ${ }^{23}$

\section{HMGB1 induces autophagy in glioma}

Studies have shown that HMGB1 promotes Beclin-1-PI3K-III complex formation by binding with Beclin-1 potentially through the methyl ethyl ketone (MEK) or extracellular signal-regulated kinase [ERK] (MEK/ERK) 1/2 pathway. ${ }^{27,28}$ Mizushima and associates stated that the phosphatidylinositide 3-kinases (PI3K) family consists of three classes: I, II, and III. They explained that, the PI3K-III activity is required for autophagic activation, while PI3KI has a negative effect on autophagy. ${ }^{29} \mathrm{He}$ and Klionsky also demonstrated that Beclin1 mobilize PI3K-III to form the Beclin-1PI3KIII complex, consequently bringing about autophagosome nucleation. ${ }^{30}$ Banzhou and colleagues showed that HMGB1 modulated autophagy by triggering the MEK/ERK1/2 pathway, while the genetic suppression of PI3K-III deprived the HMGB1-induced phosphorylation of the MEK-ERK1/2 pathway and inhibited autophagic stimulation. They proposed that MEK/ERK1/2 signaling functions as a downstream signal of PI3K-III in HMGB1induced autophagy. They also confirmed that stimulation of the MEKERK1/2 signaling pathway was linked with HMGB1-mediated formation of the Beclin-1-PI3K-III complex. ${ }^{27}$

Thorburn and associates have indicated that autophagy can regulate the pattern of dying cells by modulating the selective secretion of HMGB1, and therefore selectively controlling an influential pattern of dying cells that is usually associated with necrosis. ${ }^{31}$ They indicated that epidermal growth factor receptortargeted diphtheria toxin (DT-EGF) activates autophagy more cogently in the glioblastoma cell lines. They further argue that autophagy that is induced by DT-EGF in U87MG cells inhibits caspase activation and hence did not activate apoptosis. They demonstrated that tumor cells that are dying with increased autophagy selectively secreted the nuclear HMGB1 protein, but do so without displaying other characteristics of necrosis such as lipopolysaccharide (LDH) secretion or loss of membrane integrity such that propoidium iodide can stain DNA. ${ }^{31}$ They concluded that autophagy can activate the amount of death in response to a specific stimulus and the characteristics associated with the dying cells and that the overall biological effect of autophagy manipulation during treatment with targeted anticancer drugs like DT-EGF that kill tumor cells may depend on both aspects. ${ }^{31}$ 


\section{HMGB1 mediates glioma regression}

HMGB1 secreted from dying tumor cells might be liable for activating toll like receptor-2 (TLR-2) on dendritic cell (DC) in vivo and resultant $\mathrm{T}$ cell-dependent tumor regression (Figure 1). Curtin et al. establish that treatment of brain tumors with an adenoviral vector-thymidine kinase (Ad-TK) + ganciclovir (GCV) [AdTK (+GCV)] and adenoviruses expressing Flt3L (Ad-Flt3L) injected directly into the brain tumor microenvironment induce a systemic adaptive antiglioma immune response. They indicated that this treatment is stringently relying on the stimulation of TLR signaling on bone marrow DCs that infiltrate the tumor. They further stated that dying glioma cells secreted HMGB1 in response to infection and killing with Ad-TK $(+\mathrm{GCV})$; HMGB1 in turn induced TLR-2-dependent nuclear factor-kappaB (NF-kB) signaling and DC stimulation (Figure 1). They again confirmed that glioma-derived HMGB1 secreted from dying cells is necessary for the clonal expansion of CD8+ T cells specific for glioma antigens including the $\operatorname{Trp}_{2180-188}$ peptide $(\mathrm{H}-2 \mathrm{~Kb})$, and that the effects of HMGB1 are mediated through TLR-2 signaling on tumor-infiltrating DCs. ${ }^{32}$

Initial studies demonstrated that glioma-derived HMGB1 as the endogenous TLR-2 ligand, whose signaling is essential to evoke systemic adaptive immune-mediated glioblastoma (GBM) regression and long-term immunological memory in an intracranial glioma model. ${ }^{33}$ Curtin et al concluded that tumor-derived HMGB1 evokes endogenous TLR-2 signaling and initiates a CD8+ T cell dependent anti-GBM immune response. Hence they proposed that endogenous TLR2 ligands would also be released from isogenic glioma cells (GL261 cells) treated with Ad-TK + GCV. HMGB1-mediated TLR-2 signaling links the effects of Flt3L on the recruitment of immune cells to the brain tumor microenvironment to their capacity to induce a systemic antitumor immune response. Therefore endogenous TLR-2 ligands could play a part in tumor regression. ${ }^{32}$

\section{HMGB1 promotes angiogenesis in glioma}

Glioma-associated stromal cells like astrocytes, endothelial cells, mesenchymal cells and infiltrating inflammatory cells play a crucial function in tumorigenesis, angiogenesis, invasion and immune evasion. ${ }^{34}$ Piao et al. demonstrated that, in the midst of these cells, infiltrating microglia (MG) and macrophages (MP), (known as tumor-associated macrophages or TAMs) have recently gained attention due to their participation in glioma leakage from anti-angiogenic agents. ${ }^{35}$ Sorci and colleagues proposed that as a pattern identifying receptor, receptor for advanced glycation end products (RAGE) binds to HMGB1 that arise from damaged cells and trigger the immune system. ${ }^{36}$

Kierdorf and associates indicated that the enthrallment of RAGE by its ligands elucidate in the stimulation of multiple downstream signaling pathways which sooner or later lead to the induction of NF- $\kappa$ B, ERK1/2, p38 and signal transducer and activator of transcription (STAT3) and subsequent regulation of cytokines, chemokines, adhesion molecules, and pathways that modulate cell proliferation, survival, differentiation, migration, phagocytosis and authophagy ${ }^{37}$ (Figure 2). Also the activation of RAGE in TAMs, modifications in the manifestation of RAGE ligands may have differently wedged tumor angiogenesis in reaction to RAGE ablation in each model. van Beijnum et al. demonstrated that the K-Luc model released high levels of HMGB1 and S100A9, which intensify tumor invasion, migration and angiogenesis. ${ }^{38}$ Advanced levels of HMGB1 secreted by K-Luc gliomas may have subdue the physiological state of RAGE signaling for angiogenesis in this model. Possibly suppression of HMGB1 production in this model could be a more effective anti-angiogenic strategy than inhibiting RAGE signaling. ${ }^{34}$

\section{HMGB1 signaling pathways in gliomas}

HMGB1 is secreted by inflammatory cells and passively released from necrotic cells and actively functions as an extracellular signaling molecule during processes such as cell differentiation, tumor cell proliferation, inflammation, cell migration and tumor metastasis. ${ }^{39,40}$ Although the mechanism by which HMGB1 is involved in tumorigenesis is unclear, it is believed that activation of the Janus kinase (JAK)/signal transducer and activator of transcription (STAT) signaling pathway, which occurs through HMGB1 binding with high affinity to several receptors, including the receptor for advanced glycation end products (RAGE), toll-like receptors TLR-2, TLR-4 and TLR-9 are some of the mechanisms (Figure 1). These interactions trigger the activation of key signaling pathways involved in the regulation of cell differentiation, growth, motility and apoptosis. While some researchers have indi-

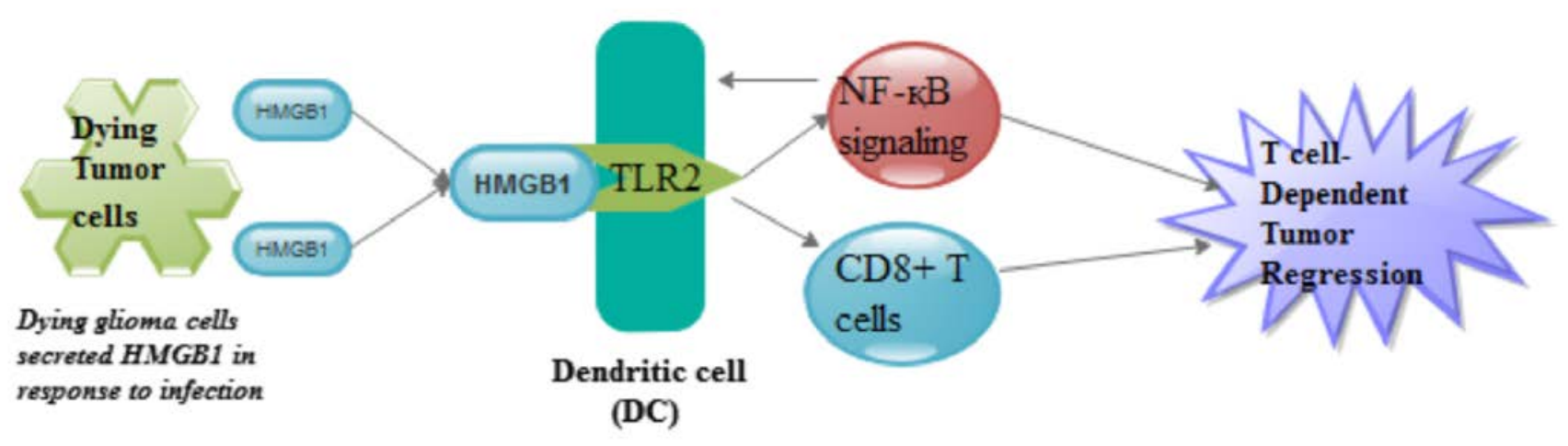

Figure 1. HMGB1 secreted from dying tumor cells in response to infection might be liable for activating TLR2 on dendritic cell (DC) in vivo and resultant $T$ cell-dependent tumor regression. 
cated that HMGB1 can over activate STAT by activating the JAK/STAT pathway and activated STAT, particularly STAT3, inhibits tumor cell apoptosis hence accelerates the cell cycle and thus leads to tumorigenesis. ${ }^{41-43}$ Others are of the view that HMGB1-RAGE interactions activate mitogen-activated protein kinase and protein kinase B signaling pathways, resulting in extracellular matrix degradation, tumor invasion and metastasis, leading to tumor development ${ }^{44}$ (Figure 2). Rosaria and colleagues noted an increase in ERK phosphorylation in their study with HMGB1 and glioblastoma cells and indicated that HMGB1-induced ERK activation and cell proliferation was effectively inhibited by PD98059 and the selective inhibitor of the mitogen-activated protein kinase (MEK) and that the functional blockade of RAGE by means of neutralizing anti-RAGE antibody strikingly prevented both the ERK phosphorylation and cell proliferation elicited by exogenous HMGB1. ${ }^{45}$ Studies have shown that HMGB1 when released into the extracellular environment unites with its high affinity receptor RAGE and upregulates RAGE expression. ${ }^{46}$

It is well known that the activated form of Ras-related C3 botulinum toxin substrate 1 (Rac1), a member of the Rho-family of small GTPases, stimulates cell migration and, as recent studies have revealed that it plays a major role in glioma invasion. ${ }^{47}$ These findings show that RAGE binding induces Racl activation and concomitantly promotes cell migration, and thus support the view of RAGE as the major receptor mediating HMGB1-dependent migration $^{48,49}$ (Figure 2). HMGB1 up-regulates the expression of MMP-9, which belongs to matrix metalloproteinases (MMPs) involved in the initiation, invasion, and metastasis of many kinds of cancers as well as glioma and gastric cancer cells which may explain its association with invasion and metastasis of glioma tumor $^{23,50}$ (Figure 1). Yu and colleagues in indicated that the hyper methylation of the CpG island upstream of miR-129-2 led to the down-regulation of miR-129-2 in glioma patients and that the demethylation of miR-129-2 by 5-aza-2'-deoxycytidine (5-AzadC) treatment increased miR-129-2 expression in glioma cells and resulted in significant inhibitory effects on cell cycle, migration, and invasion. The concluded that, the methylation status of miR129-2 may be employed as a potential biomarker in glioma. ${ }^{51}$

\section{Therapeutic potentials of HMGB1 in gliomas}

HMGB1 has been proposed as a novel target for cancer therapy, ${ }^{52}$ and further studies have shown that the validated involvement of miR-129-1/HMGB1 link in glioma cells may provide potential to use miR-129-2 and HMGB1 as therapeutic targets for glioma. Reintroducing expression of miR-129-2 in glioma cells suppresses cell growth, migration, and invasion and promotes cell apoptosis, which may provide a novel therapeutic strategy for treatment of glioma. ${ }^{51,53}$

Although no specific research data have been published concerning HMGB1 expression and functional role (and those of RAGE) in human astrocytomas, the most frequent and deadly primary intracranial tumors in adults. These tumors can show different stages of malignancy, and most low-grade tumors subsequently progress. ${ }^{45,54}$ The characteristics of high-grade tumors (including anaplastic astrocytomas and glioblastomas) are excessive proliferation, infiltrative growth, increased angiogenesis and resistance to apoptosis, which limit the success of current therapeutic approaches. Malignant gliomas also show areas of necrosis, which is a significant prognostic factor and allows the categorization of a tumor as glioblastoma. ${ }^{45,54}$

HMGB1 is also closely associated with tumor drug resistance. A previous study found that HMB1 induces autophagy, causing the cells to become resistant to chemotherapy drugs. Release of HMGB1 from dying tumor cells has been postulated to direct the immunologic response to dying cells, which determines the clinical outcome of anticancer therapies. ${ }^{32,55,56}$ Marianela and colleagues demonstrated that HMGB1 release from dying tumor cells is crucial for the efficacy of Ad-TK+GCV+Ad-Flt3L in GBMbearing rats and its blockade completely abolishes the efficacy of the therapy. HMGB1 did not seem to play a critical role in the induction of anti-GBM immunologic memory induced by the combined therapy. ${ }^{57}$ Further, the efficacy of the combined treatment is mediated by the release of the endogenous ligand HMGB1, which we have previously shown signals via TLR-2 receptors on tumorinfiltrating dendritic cells. ${ }^{32}$

HMGB1 as an endogenous TLR-2 agonist that is secreted from

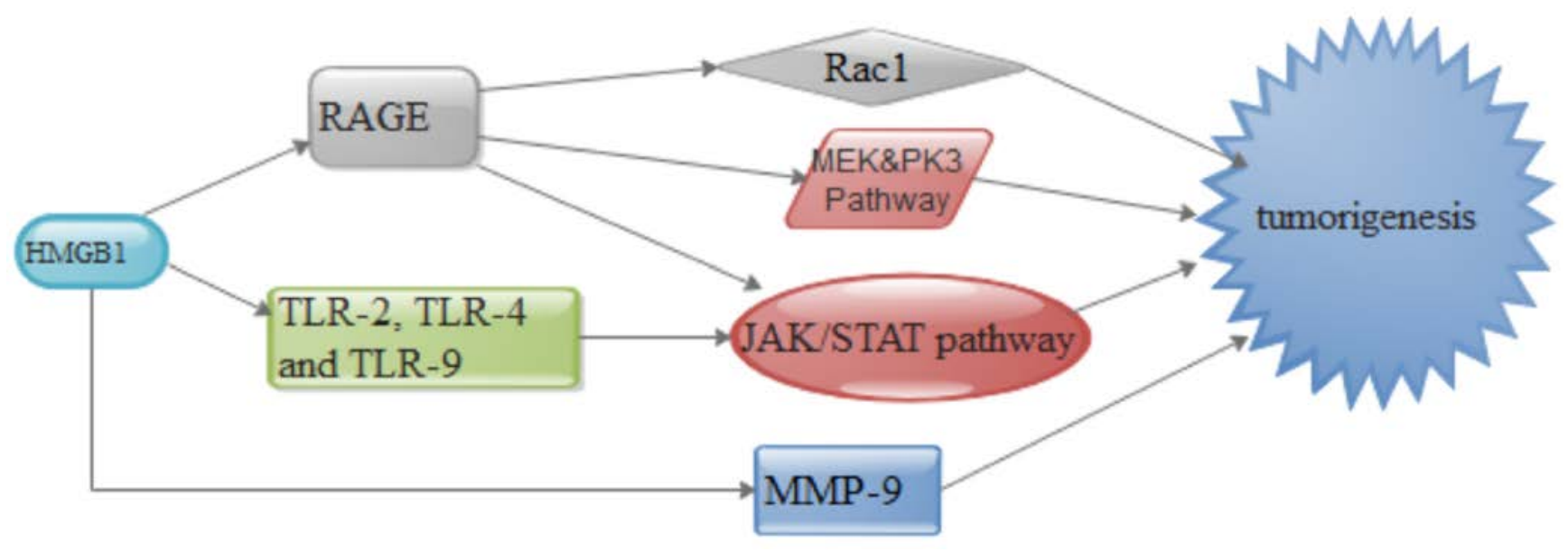

Figure 2. HMGB1 binding with high affinity to several receptors, including the receptor for advanced glycation end products (RAGE), Toll-like receptors TLR-2, TLR-4 and TLR-9 which activation of the Janus kinase (JAK)/signal transducer and activator of transcription (STAT) signaling pathway leading to tumorigenesis. HMGB1-RAGE interactions activate mitogen-activated protein kinase and protein kinase B signaling pathways leading to tumor development. RAGE binding induces Rac1 activation and concomitantly promotes tumorigenesis. HMGB1 up-regulates the expression of MMP-9 leading to tumorigenesis. 
dying tumor cells, both in vitro and in vivo in reacts to several tumor cell killing conceptualization, i.e., adenoviral vector (Ad)TK (+GCV), radiation, and temozolomide. Ad-Flt3L + Ad-TK has been proven to have potential therapeutic valve in the treatment of other solid tumors, and implying that the same molecular mechanism described initially could also be liable for the elimination of metastatic brain tumors. ${ }^{32}$

Glycyrrhizin, directly binds to and suppress HMGB 1,5 has been proven to block proliferation, migration, and angiogenesis in several tumors. ${ }^{58}$ Glycyrrhizin totally blocked TLR-2-dependent NF- $\mathrm{BB}$ stimulation by supernatants from dying meaning that HMGB1 release from GL261 cells stimulates TLR-2 signaling. Blocking HMGB1 action in vivo using glycyrrhizin or specific anti-HMGB1 neutralizing antibodies, suppressed Flt3L/TKinduced brain tumor regression. ${ }^{32}$ Glycyrrhizin does not cause the secretion of HMGB1 from apoptotic chromatin, meaning that it will not produce paradoxical pro-inflammatory responses to apoptotic cells. 59

\section{Conclusions}

Glioma-associated stromal cells like astrocytes, endothelial cells, mesenchymal cells and infiltrating inflammatory cells play a crucial function in tumorigenesis, angiogenesis, invasion and immune evasion. HMGB1 is on the upstream of the regulatory pathway and its stimulation or over-release can promote apoptosis of glioma cells. Autophagy can regulate the pattern of dying cells by modulating the selective secretion of HMGB1, and thus selectively controlling an influential pattern of dying cells that is usually associated with necrosis. HMGB1 secreted from dying tumor cells might be liable for activating TLR-2 on dendritic cell (DC) in vivo and resultant $\mathrm{T}$ cell-dependent tumor regression. Furthermore, HMGB1 may be an important prognostic marker. Therefore, treatments targeting HMGB1 are expected to become a novel therapeutic approach towards the treatment of patients with glioma.

\section{References}

1. Rees J. Diagnosis and treatment in neuro-oncology: an oncological perspective. Br J Radiol 2014 [Epub ahead of print].

2. Osman MA. Phase II trial of temozolomide and reirradiation using conformal 3D-radiotherapy in recurrent brain gliomas. Ann Trans Med 2014;2:5.

3. He J, Shan Z, Li L, et al. Expression of glioma stem cell marker CD133 and O6-methylguanine-DNA methyltransferase is associated with resistance to radiotherapy in gliomas. Oncol Rep 2011;26:1305.

4. Short SC, Giampieri S, Worku M, et al. Rad51 inhibition is an effective means of targeting DNA repair in glioma models and CD133+ tumor-derived cells. Neuro-oncol 2011;13:487-99.

5. Kang R, Zhang Q, Zeh HJ, et al. HMGB1 in cancer: good, bad, or both? Clin Cancer Res 2013;19:4046-57.

6. Akaike H, Kono K, Sugai H, et al. Expression of high mobility group box chromosomal protein-1 (HMGB-1) in gastric cancer. Anticancer Res 2007;27:449-57.

7. Völp K, Brezniceanu M-L, Bösser S, et al. Increased expression of high mobility group box 1 (HMGB1) is associated with an elevated level of the antiapoptotic c-IAP2 protein in human colon carcinomas. Gut 2006;55:234-42.

8. Ranganathan A, Gunnarsson O, Casarett D. Palliative care and advance care planning for patients with advanced malignan- cies. Ann Palliat Med 2014;3:144-9.

9. Riuzzi F, Sorci G, Donato R. RAGE expression in rhabdomyosarcoma cells results in myogenic differentiation and reduced proliferation, migration, invasiveness, and tumor growth. Am J Pathol 2007;171:947-61.

10. Ditsworth D, Zong W-X, Thompson CB. Activation of poly (ADP)-ribose polymerase (PARP-1) induces release of the proinflammatory mediator HMGB1 from the nucleus. J Biol Chem 2007;282:17845-54.

11. Zhang C-L, Shu M-G, Qi H-W, Li L-W. Inhibition of tumor angiogenesis by HMGB1 A box peptide. Med Hypothes 2008;70:343-5.

12. Dong XDE, Ito N, Lotze MT, et al. High mobility group box I (HMGB1) release from tumor cells after treatment: implications for development of targeted chemoimmunotherapy. J Immunother 2007;30:596-606.

13. Tang D, Kang R, Zeh HJ, Lotze MT. High-mobility group box 1 and cancer. Biochim Biophys Acta 2010;1799:131-40.

14. Xu X, Zhu H, Wang T, et al. Exogenous High $\square$ Mobility Group Box 1 Inhibits Apoptosis and Promotes the Proliferation of Lewis Cells via RAGE/TLR4 $\square$ Dependent Signal Pathways. Scand J Immunol 2014;79:386-94.

15. Carvajal IM, Baron RM, Perrella MA. High-mobility groupI/Y proteins: Potential role in the pathophysiology of critical illnesses. Crit Care Med 2002;30:S36-42.

16. Cebrian MJG, Bauden M, Andersson R, et al. Paradoxical Role of HMGB1 in Pancreatic Cancer: Tumor Suppressor or Tumor Promoter? Anticancer Res 2016;36:4381-9.

17. Yang H, Hreggvidsdottir HS, Palmblad K, et al. A critical cysteine is required for HMGB1 binding to Toll-like receptor 4 and activation of macrophage cytokine release. Proc Natl Acad Sci 2010;107:11942-7.

18. Yang H, Lundbäck P, Ottosson L, et al. Redox modification of cysteine residues regulates the cytokine activity of high mobility group box-1 (HMGB1). Mol Med 2012;18: 250.

19. Antoine DJ, Harris HE, Andersson U, et al. A systematic nomenclature for the redox states of high mobility group box (HMGB) proteins. Mol Med 2014;20:135-7.

20. Sims GP, Rowe DC, Rietdijk ST, et al. HMGB1 and RAGE in inflammation and cancer. Annu Rev Immunol 2009;28:367-88.

21. Zhao W, Chen Q. Effects of HMGB1 on proliferation and apoptosis of human brain glioma CD133 cells. Bratislavske lekarske listy 2014;116:480-5.

22. Martins I, Kepp O, Menger L, et al. Fluorescent biosensors for the detection of HMGB1 release. Necrosis Method Protocol 2013:43-56.

23. Zhang J, Liu C, Hou R. Knockdown of HMGB1 improves apoptosis and suppresses proliferation and invasion of glioma cells. Chinese J Cancer Res 2014;26:658-68.

24. Kostova N, Zlateva S, Ugrinova I, Pasheva E. The expression of HMGB1 protein and its receptor RAGE in human malignant tumors. Mol Cell Biochem 2010;337:251-8.

25. Jube S, Rivera ZS, Bianchi ME, et al. Cancer cell secretion of the DAMP protein HMGB1 supports progression in malignant mesothelioma. Cancer Res 2012;72:3290-301.

26. Yang GL, Zhang LH, Bo JJ, et al. Increased expression of HMGB1 is associated with poor prognosis in human bladder cancer. J Surg Oncol 2012;106:57-61.

27. Pan B, Chen D, Huang J, et al. HMGB1-mediated autophagy promotes docetaxel resistance in human lung adenocarcinoma. Mol Cancer 2014;13:1.

28. Tang D, Kang R, Livesey KM, et al. Endogenous HMGB1 regulates autophagy. J Cell Biol 2010;190:881-92.

29. Mizushima N, Yoshimori T, Levine B. Methods in mammalian 
autophagy research. Cell 2010;140:313-26.

30. He C, Klionsky DJ. Regulation mechanisms and signaling pathways of autophagy. Annu Rev Genet 2009;43:67.

31. Thorburn J, Horita H, Redzic J, et al. Autophagy regulates selective HMGB1 release in tumor cells that are destined to die. Cell Death Different 2009;16:175-83.

32. Curtin JF, Liu N, Candolfi M, et al. HMGB1 mediates endogenous TLR2 activation and brain tumor regression. PLoS Med 2009;6:e1000010.

33. Babcock AA, Wirenfeldt M, Holm T, et al. Toll-like receptor 2 signaling in response to brain injury: an innate bridge to neuroinflammation. J Neurosci 2006;26:12826-37.

34. Chen X, Zhang L, Zhang IY, et al. RAGE expression in tumorassociated macrophages promotes angiogenesis in glioma. Cancer Res 2014;74:7285-97.

35. Piao Y, Liang J, Holmes L, et al. Glioblastoma resistance to anti-VEGF therapy is associated with myeloid cell infiltration, stem cell accumulation, and a mesenchymal phenotype. Neuro-oncol 2012;14:1379-92.

36. Sorci G, Riuzzi F, Giambanco I, Donato R. RAGE in tissue homeostasis, repair and regeneration. Biochim Biophys Acta 2013;1833:101-9.

37. Kierdorf K, Fritz G. RAGE regulation and signaling in inflammation and beyond. J Leukocyte Biol 2013;94:55-68.

38. Van Beijnum J, Nowak-Sliwinska P, Van den Boezem E, et al. Tumor angiogenesis is enforced by autocrine regulation of high-mobility group box 1. Oncogene 2013;32:363-74.

39. Youn JH, Shin J-S. Nucleocytoplasmic shuttling of HMGB1 is regulated by phosphorylation that redirects it toward secretion. J Immunol 2006;177:7889-97.

40. Schlueter C, Weber H, Meyer B, et al. Angiogenetic signaling through hypoxia: HMGB1: an angiogenetic switch molecule. Am J Pathol 2005;166:1259-63.

41. Hui L, Yao Y, Wang S, et al. Inhibition of Janus kinase 2 and signal transduction and activator of transcription 3 protect against cecal ligation and puncture-induced multiple organ damage and mortality. J Trauma Acute Care Surg 2009;66:85965 .

42. Wagner K-U, Schmidt JW. The two faces of Janus kinases and their respective STATs in mammary gland development and cancer. J Carcinogen 2011;10:32.

43. Diaz T, Navarro A, Ferrer G, et al. Lestaurtinib inhibition of the Jak/STAT signaling pathway in hodgkin lymphoma inhibits proliferation and induces apoptosis. PLoS One 2011;6:e18856.

44. Ohmori H, Luo Y, Kuniyasu H. Non-histone nuclear factor HMGB1 as a therapeutic target in colorectal cancer. Exp Opin Ther Target 2011;15:183-93.

45. Bassi R, Giussani P, Anelli V, et al. HMGB1 as an autocrine stimulus in human T98G glioblastoma cells: role in cell growth and migration. J Neuro-oncol 2008;87:23-33.

46. Rauvala H, Rouhiainen A. Physiological and pathophysiological outcomes of the interactions of HMGB1 with cell surface receptors. Biochim Biophys Acta 2010;1799:164-70.

47. Salhia B, Tran NL, Symons M, et al. Molecular pathways triggering glioma cell invasion. Exp Rev Mol Diagnost 2006;6:613-26.

48. Bierhaus A, Humpert PM, Morcos M, et al. Understanding RAGE, the receptor for advanced glycation end products. J Mol Med 2005;83:876-86.

49. Huttunen H, Rauvala H. Amphoterin as an extracellular regulator of cell motility: from discovery to disease. J Intern Med 2004;255:351-66.

50. Zhang J, Kou Y-B, Zhu J-S, et al. Knockdown of HMGB1 inhibits growth and invasion of gastric cancer cells through the NF- $\kappa$ B pathway in vitro and in vivo. Int J Oncol 2014;44:126876.

51. Yang Y, Huang J-Q, Zhang X, Shen L-F. MiR-129-2 functions as a tumor suppressor in glioma cells by targeting HMGB1 and is down-regulated by DNA methylation. Mol Cell Biochem 2015;404:229-39.

52. Lu CY, Lin KY, Tien MT, et al. Frequent DNA methylation of miR-129-2 and its potential clinical implication in hepatocellular carcinoma. Genes Chromosomes Cancer 2013;52:636-43.

53. Angelopoulou E, Piperi C, Adamopoulos C, Papavassiliou AG. Pivotal role of high-mobility group box 1 (HMGB1) signaling pathways in glioma development and progression. J Mol Med 2016;94:867-74.

54. Kleihues P, Louis DN, Scheithauer BW, et al. The WHO classification of tumors of the nervous system. J Neuropathol Exper Neurol 2002;61:215-25.

55. Kepp O, Tesniere A, Schlemmer F, et al. Immunogenic cell death modalities and their impact on cancer treatment. Apoptosis 2009;14:364-75.

56. Apetoh L, Ghiringhelli F, Tesniere A, et al. Toll-like receptor 4-dependent contribution of the immune system to anticancer chemotherapy and radiotherapy. Nature Med 2007;13: 1050-9.

57. Candolfi M, Yagiz K, Foulad D, et al. Release of HMGB1 in response to proapoptotic glioma killing strategies: efficacy and neurotoxicity. Clin Cancer Res 2009;15:4401-14.

58. Musumeci D, Roviello GN, Montesarchio D. An overview on HMGB1 inhibitors as potential therapeutic agents in HMGB1related pathologies. Pharmacol Ther 2014;141:347-57.

59. Mollica L, De Marchis F, Spitaleri A, et al. Glycyrrhizin binds to high-mobility group box 1 protein and inhibits its cytokine activities. Chem Biol 2007;14:431-41. 